\title{
Leveraging User Equipment for Radio Access Network Augmentation
}

\author{
J. Pérez-Romero, O. Sallent \\ Signal Theory and Communications Department of Universitat Politècnica de Catalunya (UPC) \\ Barcelona, Spain \\ jordi.perez-romero@upc.edu, sallent@tsc.upc.edu
}

\begin{abstract}
Along the successive generations, mobile network infrastructure deployments have required continuous expansion and upgrades to respond to the ever increasing traffic demand. At the same time, user equipment (UE) has evolved with the introduction of powerful and advanced communication and computational capabilities. Moreover, several industrial initiatives are promoting the principles of RAN disaggregation through the split of RAN functionalities among multiple interoperable components in contrast to traditional monolithic solutions. Embracing these trends, this paper presents the vision of a Beyond 5G (B5G) scenario where the UE takes a more active role in the communication service provisioning and is used to augment the RAN infrastructure as a source of distributed capacity and network intelligence. This is expected to bring benefits for Mobile Network Operators by reducing the number of base stations to deploy and by providing the RAN with a new degree of flexibility for smartly and dynamically adapting the RAN to the specific needs. In this direction, after discussing the main enabling technologies of the envisaged RAN augmentation concept, this paper presents a simulation-based assessment to quantify the performance improvements and the achievable savings in terms of infrastructure deployment.
\end{abstract}

Keywords- Beyond 5G, Radio Access Network, User Equipment, Machine Learning, Federated Learning, UE-tonetwork relaying.

\section{INTRODUCTION}

Over the 40 years of its history, the mobile network infrastructure has been continuously expanding and upgraded with new technologies to the point that it has already become the largest technology platform in human history, with around 8.000 million mobile subscriptions worldwide by 2020 and reaching subscription penetration as high as $136 \%$ of population in Central and Eastern Europe [1]. The Radio Access Network (RAN) equipment accounts for the highest portion of this infrastructure, with millions of macro, micro and small cell base stations deployed worldwide. Fuelled primarily by the surge in video traffic, mobile network operators (MNOs) are forced to respond promptly with decisive capacity scaling on their deployments to face high traffic demand (e.g., mobile data traffic grew by $51 \%$ between 2019 and 2020 and projections for the coming years are simply overwhelming [1]).

The insatiable appetite for mobile connectivity, prompts massive capital expenditure (CAPEX) on network improvements (e.g., deployment of RAN infrastructure) as consumer demand is dictating the pace. However, dwindling average revenues per user, market saturation and intensifying competition make the MNOs seeing their finances stretched. To alleviate this pressure, expectations have been created around the Fifth Generation (5G) as the key to unlock new sources of revenue, capitalizing on emerging vertical markets. Nevertheless, MNOs need to find not only new revenue streams but also new and creative ways of managing and deploying their $5 \mathrm{G}$ and beyond infrastructure towards costeffectiveness.

Likewise, from the user equipment (UE) side, we have also witnessed an unprecedented technological evolution leading to UEs with very powerful and advanced communication and computational capabilities. This is visible both in the form of personal use devices, such as smartphones and high-end wearables, as well as equipment integrated in other platforms such as cars and drones.

Embracing the abovementioned trends, this paper envisions a Beyond $5 \mathrm{G}(\mathrm{B} 5 \mathrm{G})$ scenario where the UE is also exploited to augment the RAN infrastructure as a source of distributed capacity and network intelligence. In other words, we envision the idea of UEs taking a more active role in network service provisioning as one of the pillars to ground future network evolution, to the extent that the very notion of a cell will have to be rethought as UEs will be able to actively complement the RAN infrastructure.

The engagement of the UEs in support of service provisioning is expected to positively impact MNOs in several facets. On the one hand, important CAPEX savings can be achieved as the involvement of UEs will eventually allow reducing the number of fixed base stations to deploy. On the other hand, the involvement of UEs will empower the RAN with a new degree of flexibility, by e.g., enhancing the performance in mitigating objects' obstructions in outdoor millimetre wave deployments, augmenting capacity in highdensity areas, providing coverage extension in outdoor/indoor areas, or improving resilience. For example, UEs at vehicles temporarily parked in popular locations could provide coverage and connectivity to other neighbour devices outside these vehicles. Thanks to better Radio Frequency (RF) and antenna capabilities, they could get better macrocell coverage than nearby UEs, thus providing them with a better link to the cellular network. Other example use cases could consider connectivity to devices inside a vehicle, such as a user inside a taxi, bus or tram, UEs placed at motorized balloons or drones at stadiums providing coverage to the attendees or a camera from a motorbike following a sporting race using a UE from an alongside vehicle to relay traffic.

In this context, this paper promotes the vision of a dynamic and adaptable RAN, where the performance footprint provided by a fixed B5G RAN infrastructure is smartly augmented with the networking, computing and storage resources brought by UEs. Although some of the enabling technologies of this concept, such as the relays, have been in place for years, the authors claim that moving from the concept to its realization embraces the development of new features in B5G systems that require substantial research challenges. These span from top level service layer capabilities for MNOs and UE owners to interact with each other to settle the conditions for using the UE in the RAN augmentation, down to the necessary management and control layer capabilities for exploiting the connectivity brought by 
the UEs. Beyond technical aspects, developing the business perspective (e.g., through devising proper incentives for those users that contribute their UEs) is required, considering the impact of the relaying on the power consumption, due to the cost on energy availability for a UE that acts as relay.

In turn, in views of the widespread exploitation of Artificial Intelligence (AI) and Machine Learning (ML) in the $\mathrm{B} 5 \mathrm{G}$ era, the integration of the UEs as part of an end-to-end connected network intelligence framework is relevant for materializing true context awareness and ubiquitous AI, which become an essential component to be embedded in the augmented RAN's system design. In this way, the paradigm shift relies on UEs extracting information and knowledge of the environment for better network operation, enabling UE-toUE and UE-to-network knowledge transfer and facilitating the execution of $\mathrm{AI} / \mathrm{ML}$ models in the UE for smart networking and for smartly selecting the UEs to enhance the connectivity of other UEs.

With all the above, these paper has a two-fold objective. On the one hand, the paper presents the baseline enabling technologies and outlines the supporting architecture to materialize the concept together with the description of the key novel features. This is presented in Section II. On the other hand, and before moving towards system design (which obviously exceeds by far the scope of a paper), the paper focuses on assessing the benefits that the augmented RAN concept can bring. This is presented in Section III, in terms of infrastructure savings. Motivated by the remarkable gains that can be achieved, Section IV summarises the conclusions and points out the future work towards the development of the different system components.

\section{ENABLING TECHNOLOGIES}

The advent of $5 \mathrm{G}$ systems has arrived with the emergence of a set of technologies, such as Network Function Virtualization (NFV), Multi-access Edge Computing (MEC), service-based architectures or Software Defined Networks (SDN), which enable the disaggregation of the RAN infrastructure through the split of the RAN functionalities among different interoperable hardware and software components and the definition of open interfaces. Industrial initiatives such as the O-RAN Alliance (https://www.oran.org/), the Telecom Infra Project (TIP) (https://telecominfraproject.com/) or the Small Cell Forum (https://www.smallcellforum.org/) are promoting these principles in contrast to the traditional use of proprietary, closed systems and monolithic solutions. The augmented RAN concept discussed in this paper brings this disaggregation trend one step forward by effectively integrating the UE as another interoperable component of the RAN. Then, in addition to the abovementioned relevant technologies, the following sections discuss the key enabling technologies to ensure the connectivity between UEs, to integrate the UE as part of an end-to-end connected network intelligence framework and the possible supporting architectures for realizing the concept.

\section{A. UE-to-network relaying}

The option of deploying fixed relay stations for providing a cost-effective way to extend the coverage and capacity in a cellular network has been well analysed in the literature (see e.g., [2]). Some studies have even considered the possibility of mobile relays for efficient support of devices that move as a group on e.g., a bus or a train (see e.g., [3]). Similarly, the vision of moving networks to match dynamic user demand with network access supply was described in [4]. Unfortunately, the commercial adoption of relays in previous systems such as Long Term Evolution (LTE) was not successful mainly because the LTE spectrum is too expensive to be used for backhauling and because the small cell deployments did not reach the anticipated potential at that time [5]. However, the situation is changing for $5 \mathrm{G}$ and beyond systems, due to the much larger bandwidths available in millimetre wave spectrum bands, the worse propagation conditions at these bands and the need of densified deployments to fulfil the expected data rate requirements.

For these reasons, the Third Generation Partnership Project (3GPP) has recently introduced a new relaying technology referred to as Integrated Access and Backhaul (IAB), which provides an alternative to fibre backhaul by extending 5G New Radio (NR) to support wireless backhaul [6][7]. It is based on an IAB donor that provides an interface with the core network and wireless backhauling functionality to IAB nodes, which create cells of their own and appear as normal base stations to UEs connecting to them. The IAB node connects to the network using the same initial-access mechanism as a terminal and once connected, it receives the necessary configuration from the donor node. Additional IAB nodes can connect to the network through the cells of an IAB node, thereby enabling multi-hop wireless backhauling. IAB functionality as specified by $3 \mathrm{GPP}$ in [7] assumes fixed IAB nodes. The recent study item [8] considers the possibility of having relays mounted on vehicles, which could be seen as the extension of IAB to incorporate mobility. Then, leveraging this feature, this paper considers a further evolution of the IAB concept in which the IAB capabilities are brought to the UEs and in which UEs with support of IAB functionality could be dynamically activated to become RAN nodes and conveniently exploited to relay traffic from nearby UEs.

Another candidate technology for UE-to-network relaying is the use of Device-To-Device (D2D) communications [9], initially introduced by 3GPP under the Proximity Services (ProSe) concept to address public safety-related use cases and later on extended to vehicle-to-vehicle communications. For D2D operation, a new PC5 interface between UEs was defined in LTE and 5G NR together with a radio link arrangement for direct transmissions between devices, denoted as sidelink. The support of proximity services including also commercial applications in 5G has been conducted in a Release 17 study item [10] to identify the required enhancements to the $5 \mathrm{G}$ system architecture, including the support of UE-to-network relay, in which a UE relays the traffic of another UE to/from the network in a two-hop communication. This capability has been included among the connectivity models of [11] in Release 18, stating that a UE can connect to the network directly, indirectly using another UE as a relay or using both direct and indirect connections and identifying different scenarios for the use of relay UEs (in home, smart farming, smart factories or public safety), together with requirements and key performance indicators. UE-to-network relay can be provided utilizing in-band $\mathrm{D} 2 \mathrm{D}$, in which the $\mathrm{D} 2 \mathrm{D}$ link and the cellular link use the same spectrum, and out-of-band D2D, in which the D2D link and the cellular links use different frequency bands or even different technology (e.g. Wi-Fi) [9].

In addition to the IAB and D2D technologies, another option for the UE-to-network relaying would be the use of a relay operating only at the $\mathrm{PHY} / \mathrm{RF}$ layer. In this direction, $3 \mathrm{GPP}$ is recently considering the use of so-called smart repeaters as a potential study item for Rel. 18 [12]. These are 
RF repeaters in which side control information becomes available including downlink/uplink splits or transmit/receive beam information.

\section{B. AI/ML architectural frameworks and federated learning}

The augmented RAN concept envisages the UE integrated in a connected network intelligence framework, spanning both the network and the end-user devices. For this purpose, it is possible to leverage and expand the architectural frameworks defined by the International Telecommunication Union (ITU) in [13], which provides a set of overarching requirements and high-level architectural components for AI/ML deployment in future networks, and by the O-RAN Alliance [14], which provides a more specific architecture for AI/ML deployment on the RAN. Currently, the role of the UE in both frameworks basically serves as a producer of data, actor or subject of action. However, the UE has not been so far considered as a potential host for ML model training and inference as envisioned here. Indeed, the reality is that today UEs are rapidly evolving to include multiple hardware and software components that turn them into powerful $\mathrm{AI} / \mathrm{ML}$ processing devices, such as the Qualcomm AI engine [15].

Different ML models are envisaged to be hosted at the UE supporting different decision-making functions, such as the decision on when to activate/deactivate the relaying capabilities. Examples include an activity forecasting model that anticipates the data sessions and data requirements of the UE, a model that learns locations where the UE uses to remain stationary for some time or a context learning model that characterizes the operational environment of the UE in terms of e.g. the nearby UEs that would benefit from the UE acting as relay. The context learning embraces wider functionalities, as it can exploit mobility and coverage forecasting models to anticipate e.g. bad coverage situations due to e.g. blocking obstacles, associate this with historical data and trigger the appropriate decisions to prevent service interruptions. For all the ML models hosted at the UE, while the ML model inference can remain local at each UE, the use of federated learning [16] is identified as a useful tool for efficient ML model training. In federated learning, each UE trains a local version of a model based on its own data and sends it to a model aggregator, which combines the received models and generates a new upgraded version to be further trained locally by the UEs [17]. In this way, UEs will be able to collectively learn ML models in a fast and efficient way.

\section{Supporting architecture}

From an architectural perspective, to support the proposed concept we can take as a reference the O-RAN architecture defined by O-RAN Alliance [18] to complement 3GPP 5G standards with a foundation of virtualized network elements, white-box hardware and standardized interfaces that fully embrace the core principles of intelligence and openness. At the RAN infrastructure side, this architecture considers a disaggregated RAN where the radio protocol stack of a base station (e.g. gNB) is split and distributed between the O-RAN Radio Unit (O-RU), which hosts the RF processing and the lower part of the physical layer functionality, the O-RAN Distributed Unit (O-DU), which is in charge of the rest of physical layer processing, Medium Access Control (MAC) and Radio Link Control (RLC), and the O-RAN Central Unit (O-CU), which hosts the upper layers of the radio protocol stack, i.e. Radio Resource Control (RRC), Packet Data Convergence Protocol (PDCP) and Service Data Adaptation Protocol (SDAP). On top of these RAN nodes the near-real- time RAN Intelligent Controller (near-RT RIC) serves as the brain of the RAN and deals with different Radio Resource Management (RRM) functions needed for overall RAN operation.

The Service Management and Orchestration (SMO) provides support for Fault, Configuration, Accounting, Performance and Security (FCAPS) management of the different elements of the O-RAN (i.e. near-RT RIC, O-CU, ODU, O-RU) through the $\mathrm{O} 1$ interface. The SMO also includes the non-real-time RAN Intelligent Controller (non-RT RIC) that supports intelligent RAN optimization by providing policy-based guidance, model management and enrichment information to the near-RT RIC via the A1 interface [18] and its time scale of operation is larger than $1 \mathrm{~s}$, in contrast to the near-RT RIC that operates at shorter time scales. To support its functionalities, the non-RT RIC includes different modular applications, referred to as rApps, which can e.g. drive content across the A1 interface, recommend content for the $\mathrm{O} 1$ interface to other SMO functions, generate information to be used by other rApps, etc.

At the SMO side, it is envisaged that new functions are required as part of the non-RT RIC, running as rApps, for supporting the RAN augmentation concept. Examples of these functions are related to the decision on the activation/deactivation of the relaying capabilities at a given UE or its configuration after its activation (e.g. deciding its operating frequency, the transmit power, etc.). Concerning the decision making process for the activation/deactivation of the relaying capabilities, it is envisaged that the decision would be taken, in a first stage, at the UE based on its own local information (e.g. battery status, propagation and interference conditions, etc.) and then this decision would be corroborated in case at the non-RT RIC based on a global view of the network (e.g., knowledge of other UEs in the surroundings that are already activated). This distributed approach appears to be advantageous with respect to a centralized decision at the network, because the latter becomes less scalable because it would require a decision model for a multiplicity of candidate UEs to be controlled by the network. ML and, more specifically, deep reinforcement learning (DRL) is envisaged to become a candidate solution for this function thanks to the capability of learning how to make decisions in problems that depend on a multiplicity of inputs taking a wide range of possible values.

\section{Assessment of Potential Benefits}

To assess the potential improvements of the considered framework, a set of system level simulations have been performed in a realistic urban scenario of $700 \times 700 \mathrm{~m}$ in Barcelona city, encompassing an area with different streets, avenues and a park, as shown in Fig. 1(a). The total population density is 43300 inhabitants $/ \mathrm{km}^{2}$, and a mobile network operator with $20 \%$ market penetration has been considered, leading to a density of $8660 \mathrm{UEs} / \mathrm{km}^{2}$. It is assumed that, during a simulation, $40 \%$ of the UEs are pedestrian, $50 \%$ are stationary and $10 \%$ are vehicular. Pedestrian UEs move at 3 $\mathrm{km} / \mathrm{h}$ along the sidewalks of the streets with $20 \%$ probability of changing direction at an intersection or following a random walk model around the park areas in which the UE maintains the same direction for an exponentially distributed time with average $10 \mathrm{~s}$ and makes random direction changes in a range $\left(+45^{\circ},-45^{\circ}\right)$ with respect to the current direction. Stationary UEs remain static throughout a simulation and can be placed either outdoors at a sidewalk or a park or indoors at any of the 


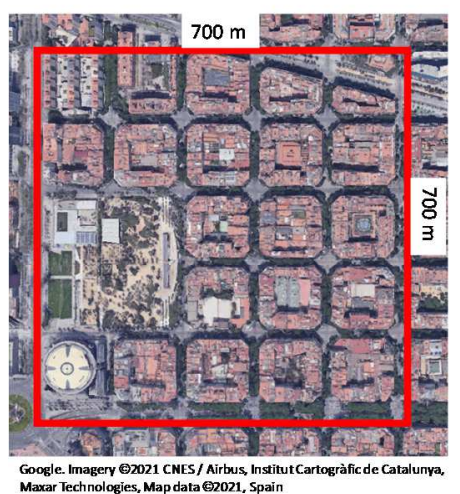

(a)

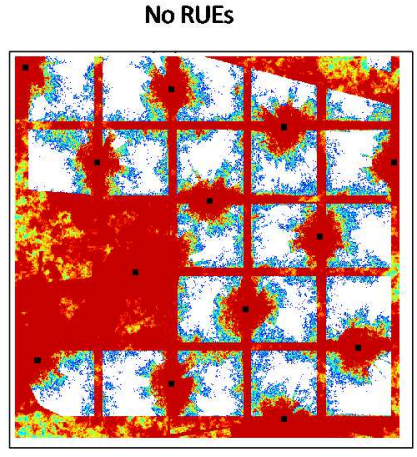

(b)

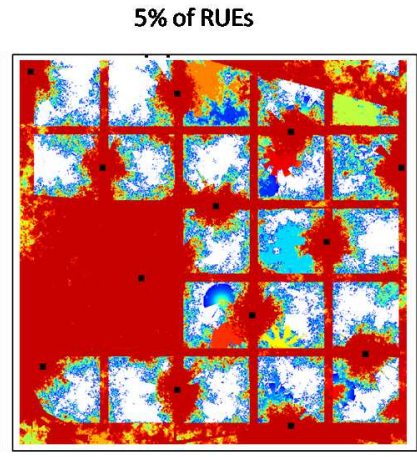

(c)
$20 \%$ of RUES

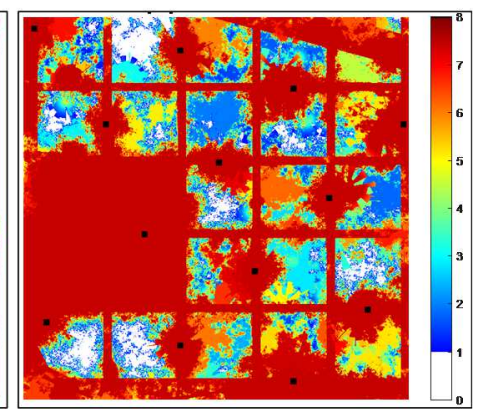

(d)

Fig. 1. Scenario under study (a) and spectral efficiency (in b/s/Hz) at the ground floor without RUEs (b), with 5\% of stationary UEs acting as RUEs (c) and with $20 \%$ of stationary UEs acting as RUEs (d).

floors of a building. Vehicular UEs move along the streets at $30 \mathrm{~km} / \mathrm{h}$ with $25 \%$ probability of changing the street at an intersection. All UEs generate data sessions following a Poisson distribution with rate 1 session/h/UE and exponential duration with average $300 \mathrm{~s}$.

As a reference $5 \mathrm{G}$ NR deployment it is assumed that the scenario is covered by 13 outdoor microcell base stations (BS) placed at the black dots of Fig. 1(b) corresponding to an intersite distance (ISD) of approximately $200 \mathrm{~m}$, as considered by the Urban Microcell (UMi) scenario of 3GPP TR 38.901. Antenna height is $10 \mathrm{~m}$, frequency is $26 \mathrm{GHz}$ and the total transmitted power is $25 \mathrm{dBm}$ over a total bandwidth of 100 $\mathrm{MHz}$. Beamforming with ideal beam steering is assumed with an antenna gain of $26 \mathrm{~dB}$ for the microcells and $10 \mathrm{~dB}$ for the UEs. UE height is $1.5 \mathrm{~m}$ on top of height of the floor where they are located. Seven floor buildings are considered with 3.5 $\mathrm{m}$ floor height. Only the downlink direction is considered, and the noise figure of the UE receiver is $9 \mathrm{~dB}$. The propagation follows the UMi model of 3GPP TR 38.901 with outdoor-tooutdoor and outdoor-to-indoor losses and 2D-spatially correlated shadowing. A UE is served by the microcell with the highest signal-to-noise ratio (SNR). It is assumed that interference among cells is negligible due to the large amount of spectrum available in the $26 \mathrm{GHz}$ band, which facilitates deployments with low frequency reuse, and to the interference coordination that can be achieved when transmitting with narrow antenna beams.

Fig. 1(b) shows a map of the spectral efficiency obtained at the ground level of the scenario. It is computed using the Shannon formula bounded by the limit of $7.4063 \mathrm{~b} / \mathrm{s} / \mathrm{Hz}$ corresponding to the maximum modulation and coding scheme of 5G NR defined in 3GPP TS 38.214. It is assumed that a minimum spectral efficiency of $1 \mathrm{~b} / \mathrm{s} / \mathrm{Hz}$ is required for proper service provisioning. Thus, UEs with spectral efficiency lower than $1 \mathrm{~b} / \mathrm{s} / \mathrm{Hz}$ are assumed to be in outage. The pixels in outage are depicted in white in Fig. 1(b). It is observed that the outdoor coverage is overall very good, but the indoor coverage is very poor, with many regions in outage. Although not shown in the figure, this effect is exacerbated in higher floors of the buildings.

A certain fraction of the stationary UEs are assumed to be activated as Relay UE (RUE) for relaying traffic to other pedestrian and stationary UEs, while vehicular UEs are served by the microcells. RUEs transmit at $3.5 \mathrm{GHz}$ with the same power and bandwidth than the microcells and with antenna gain $3 \mathrm{~dB}$. Only stationary UEs with spectral efficiency larger than $1 \mathrm{~b} / \mathrm{s} / \mathrm{Hz}$ are considered as RUE candidates. The UE-to-
UE propagation model is taken from [19] but including the outdoor-to-indoor propagation losses of the UMi model and an additional loss of $21 \mathrm{~dB}$ per floor when UEs are located in different floors of the same building. Perfect interference coordination among RUEs is assumed. Moreover, since the direct and the relay links operate in different bands, it is assumed that, when a UE is served by a RUE, the obtained spectral efficiency is the minimum between the one of the microcell-RUE link and the RUE-UE link.

Fig. 1(c) presents an example of the spectral efficiency maps at the ground level when $5 \%$ of the stationary UEs are randomly selected to be RUEs, and Fig. 1(d)shows an example when $20 \%$ of stationary UEs act as RUEs. It is observed that, by activating more RUEs the spectral efficiency is progressively improved and the outage areas are substantially reduced, particularly inside the buildings. It is worth mentioning that further improvements could be expected with a smart selection of the most convenient UEs to be activated, rather than the random approach considered in this example.

As two sides of the same coin, the observed coverage footprint improvement can also be seen in terms of infrastructure savings. In this respect, Fig. 2 plots the total outage probability obtained with the reference deployment of 13 microcell BSs and with smaller deployments of 6 BSs (average ISD of around $300 \mathrm{~m}$ ) and 3 BSs (average ISD of around $370 \mathrm{~m}$ ). Each point in the figure is the outage probability obtained for all the UEs in the scenario after executing a total of 50 dynamic simulations of $10000 \mathrm{~s}$ duration, changing randomly the distribution of stationary UEs and RUEs from one simulation to another. Clearly, Fig. 2 shows that the outage probability reduces progressively when increasing the fraction of RUEs, thus allowing to quantify the improvements observed in Fig. 1. Moreover, taking as a reference the baseline deployment of $13 \mathrm{BSs}$ without RUEs, for which the outage probability is $16.5 \%$, the red dotted line of Fig. 2 reflects that the same outage probability is obtained approximately with a deployment of 6 BSs and $6 \%$ of stationary UEs being RUEs and with a deployment of only 3 BSs and $16 \%$ of RUEs. That is, engaging around $20 \%$ of stationary UEs in the considered scenario as RUEs would allow reducing the deployed fixed RAN infrastructure in around $75 \%$, thus achieving substantial CAPEX savings for the MNO.

To assess the effect of reducing the number of deployed microcells on the performance of the different UE types, Fig. 3 depicts the average spectral efficiency experienced by the 
pedestrian, stationary and vehicular UEs with the deployment of $3 \mathrm{BSs}$ as a function of the fraction of RUEs. Outdoor UEs (either pedestrian or stationary) achieve a good average spectral efficiency of about $5 \mathrm{~b} / \mathrm{s} / \mathrm{Hz}$ without RUEs due to favourable propagation conditions. Then, a remarkable gain of about $40 \%$ is obtained with $20 \%$ of RUEs. Nonetheless, the highest benefit is obtained by the indoor stationary UEs, which experience a very poor spectral efficiency of only 0.6 $\mathrm{b} / \mathrm{s} / \mathrm{Hz}$ without RUEs and it improves in $226 \%$ with $20 \%$ of RUEs and in $425 \%$ with $80 \%$ of RUEs. Finally, vehicular UEs, which are always served by the microcells, also experience a satisfactory performance even if the number of BSs has been reduced with respect to the reference deployment of 13 BSs.

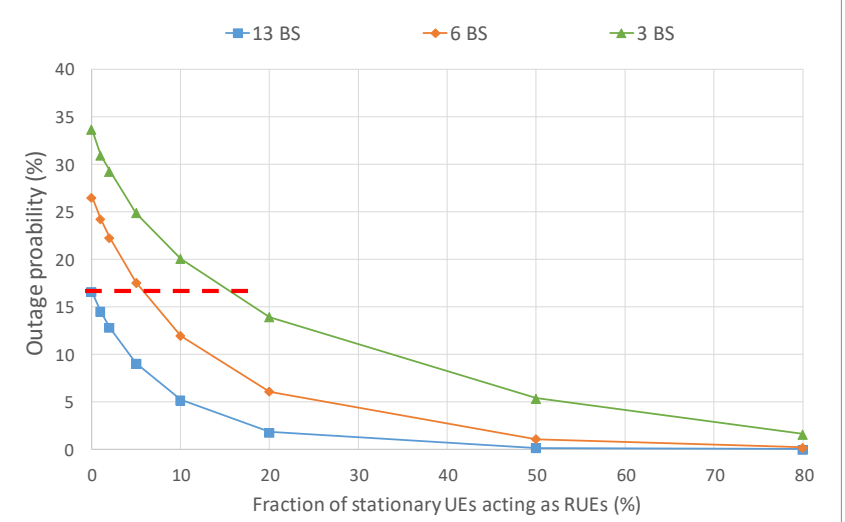

Fig. 2. Outage probability as a function of the fraction of stationary UEs acting as RUEs

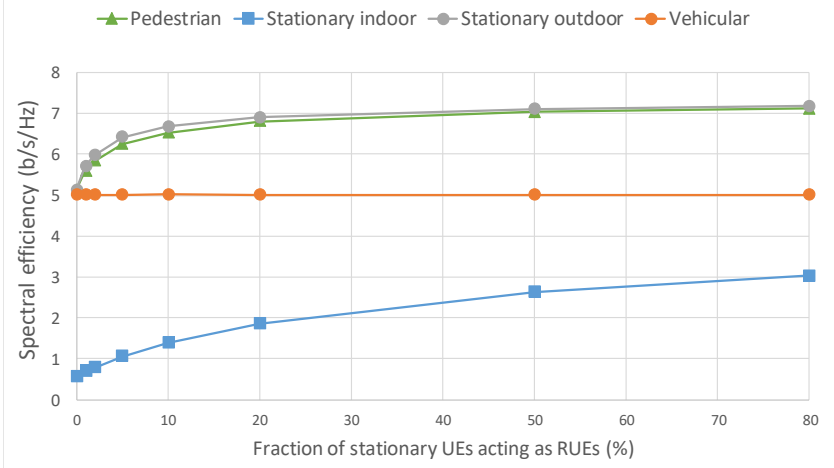

Fig. 3. Average spectral efficiency for the different UE types with the deployment of 3 BSs

\section{CONCLUSIONS AND FUTURE WORK}

The paper has presented a vision of a B5G scenario in which the UE is used to augment the RAN infrastructure as a source of distributed capacity and network intelligence. It is sustained on UE-to-network relaying capabilities and on a connected network intelligence framework in which the UEs extract information of the environment and contribute to the training and execution of $\mathrm{AI} / \mathrm{ML}$ models for smart networking. This is expected to bring benefits for mobile network operators in terms of CAPEX reductions and more flexible and adaptive RANs. Specifically, the paper has shown through system-level simulations in a realistic urban scenario that engaging around $20 \%$ of the stationary UEs to act as relays allows reducing the deployed fixed RAN infrastructure in around $75 \%$. Moreover, spectral efficiency improvements in the order of $40 \%$ for outdoor users and over $200 \%$ for indoor users are observed for $20 \%$ of RUEs with respect to the case without RUEs. Motivated by these promising results, the next steps will target the specification of the functional architecture for the augmented RAN as well as the identification and development of the AI/ML models that will support the operation of the network, with particular focus on the decision making for activating/deactivating the relaying capabilities at the UEs.

\section{ACKNOWLEDGMENT}

This paper is part of ARTIST project (ref. PID2020115104RB-I00) funded by MCIN/AEI/10.13039/ 501100011033. The authors would like to thank Dr. Ramon Ferrús, Dr. Behnam Shariati and Prof. Haris Gačanin for the insightful discussions that have helped us to give shape to the concepts presented in this paper.

\section{REFERENCES}

[1] Ericsson Mobility Report, February, 2021.

[2] J. Sydir, R. Taori, "An Evolved Cellular System Architecture Incorporating Relay Stations", IEEE Communications Magazine, June 2009

[3] R. Balakrishnan, X. Yang, M. Venkatachalam, I. F. Akyildiz, "Mobile Relay and Group Mobility for 4G WiMAX Networks", IEEE Wireless Communications and Networking Conference, 2011

[4] S. Andreev, V. Petrov, M. Dohler, H. Yanikomeroglu, "Future of UltraDense Networks Beyond 5G: Harnessing Heterogeneous Moving Cells", IEEE Communications Magazine, June 2019

[5] O. Teyeb, A. Muhammad, G. Mildh, E. Dahlman, F. Barac, B. Makki, "Integrated Access Backhauled Networks", IEEE 90th Vehicular Technology Conference (VTC-2019 Fall), 2019.

[6] M. Polese, et al. "Integrated Access and Backhaul in $5 \mathrm{G}$ mmWave Networks: Potential and Challenges", IEEE Communications Magazine, March, 2020.

[7] 3GPP TS 38.300 v16.7.0, "NR; NR and NG-RAN Overall Description; Stage 2 (Release 16)", September, 2021

[8] 3GPP TR 22.839 v18.0.0, "Study on Vehicle-Mounted Relays; Stage 1 (Release 18)", September, 2021.

[9] A. Asadi, Q. Wang, and V. Mancuso, "A survey on device-to-device communication in cellular networks," IEEE Comm. Surveys Tuts., vol. 16, no. 4, pp. 1801-1819, Fourth Quart., 2014.

[10] 3GPP TR 23.752 v17.0.0, "Study on System enhancement for Proximity based Services (ProSe) in the 5G System (5GS) (Release 17)", March, 2021.

[11] 3GPP TS 22.261 v18.3.0, "Service requirements for 5G system; Stage 1 (Release 18)", June, 2021.

[12] Qualcomm, "NR Smart Repeaters for Rel-18", 3GPP TSG RAN Rel18 workshop, June 28-July 2, 2021.

[13] Recommendation ITU-T Y.3172, "Architectural framework for machine learning in future networks including IMT-2020”, June 2019.

[14] O-RAN Working Group 2, "AI/ML workflow description and requirements v01.00”, December, 2019

[15] Qualcomm, "Making AI ubiquitous", February, 2020, https://www.qualcomm.com/media/documents/files/making-aiubiquitous.pdf (Accessed July 2021)

[16] Y. Liu, X. Yuan, Z. Xiong, J. Kang, X. Wang, D. Niyato, "Federated Learning for 6G Communications: Challenges, Methods, and Future Directions", China Communications, September, 2020

[17] T. Li, A. K. Sahu, A. Talwakar, V. Smith, "Federated Learning. Challenges, methods and future directions", IEEE Signal Processing Magazine, May,2020

[18] O-RAN Alliance, "O-RAN Architecture Description v4.0", March, 2021.

[19] Siemens AG, “TDD UE-UE Interference Simulations", R4-030189 document of the 3GPP TSG-RAN Working Group 4 meeting \#26, February, 2003. 